\title{
VANTAGENS E LIMITAÇÕES DE ALGUNS INSTRUMENTOS DE RASTREIO COGNITIVO USADOS NO BRASIL NA AVALIAÇÃO DA DEMÊNCIA
}

\section{REVISÃO BIBLIOGRÁFICA}

REIS, Edson Mário dos ${ }^{1}$

REIS, Edson Mário dos. Vantagens e limitações de alguns instrumentos de rastreio cognitivo usados no brasil na avaliação da demência. Revista Científica Multidisciplinar Núcleo do Conhecimento. Ano 03, Ed. 11, Vol. 06, pp. 22-48 Novembro de 2018. ISSN:2448-0959, Link de acesso: https://www.nucleodoconhecimento.com.br/psicologia/vantagens-e-limitacoes, DOI: 10.32749/nucleodoconhecimento.com.br/psicologia/vantagens-e-limitacoes

\section{RESUMO}

Introdução: Com a população idosa crescente no Brasil, as doenças que causam demência tornaram-se as enfermidades neuropsiquiátricas de maior prevalência na terceira idade. O diagnóstico etiológico é baseado em exames laboratoriais, neuroimagem e perfil neuropsicológico característico. Há dificuldades em identificar demência precoce somente através de avaliação clínica e exames médicos de rotina, o que compromete o diagnóstico diferencial. Portanto, faz-se necessário utilizar, tanto os instrumentos de rastreio cognitivo usados no início do processo diagnóstico, quanto os demais instrumentos neuropsicológicos utilizados nas investigações mais amplas. Sabe-se que existe a necessidade de mais informações sobre as vantagens e limitações dos instrumentos utilizados no rastreio cognitivo que facilite a seleção daqueles que mais atendam a demanda do avaliado e do avaliador. Objetivos: Identificar e descrever as vantagens e limitações de alguns dos

\footnotetext{
${ }^{1}$ Psicólogo Clínico e Neuropsicólogo. Doutorando em Psicologia pela Universidade de Ciencias Empresariales Y Sociales - UCES - Buenos Aires - Ar.
}

$\mathrm{RC}: 22961$

Disponível em: https://www.nucleodoconhecimento.com.br/psicologia/vantagens-elimitacoes 
instrumentos de rastreio cognitivo mais utilizados no Brasil na valiação da demência. Metodologia: Pesquisa bibliográfica em bases eletrônicas e livros, artigos, revistas, periódicos, dissertações e teses com publicações em português e inglês, entre outros, por meio de buscas nas bases de dados BVS Psicologia (DeCS), LILACS, SCIELO, PsyINFO, PubMed. Critérios de exclusão: artigos e livros que não atendam os objetivos da pesquisa. Resultados: 1) Disponibilidade de um quadro para consulta das características psicométricas dos instrumentos de avaliação neuropsicológica. 2) Existem limitações características de cada teste de rastreamento cognitivo que de certa forma o restringe em determinado contexto. 3) É importante ter cautela na utilização desses instrumentos, contudo, existem também muitas vantagens entre os testes. O ideal é que os instrumentos escolhidos apresentem acurácia, níveis de corte adequados por idade e/ou escolaridade e fidedignidade.

Palavras chave: Envelhecimento, Demência, Neuropsicologia, Rastreamento, Cognitivo.

\section{INTRODUÇÃO}

Sabe-se que existem dificuldades no tocante à escolha e seleção de instrumentos de rastreio cognitivo para montar um processo de Avaliação Cognitiva (AC) e/ou uma Avaliação Neuropsicológica (AN) mais ampla. Mesmo porque, existem características peculiares de cada instrumento, dos vários que existem no mercado, que dificultam ainda mais tal escolha. Diante disso, percebeu-se a necessidade de informações sobre as vantagens e limitações de instrumentos utilizados no rastreio cognitivo que possam facilitar a seleção daqueles que mais atendam a demanda do avaliado e do avaliador, para que hajam resultados mais acurados das testagens e, consequentemente, possibilidade de fechamento de uma hipótese diagnóstica mais fidedigna.

A proposta desse artigo foi de atender essa demanda e, a partir disso, produzir e disponibilizar para consulta no (QUADRO-2) com referências de alguns dos 
instrumentos de rastreio cognitivo mais utilizados no Brasil na $\mathrm{AN}$ de idosos com suspeita de demência; e ainda, apresentar os instrumentos de rastreio cognitivo com suas características peculiares, onde, ao final, o leitor terá a possibilidade de saber além das características de cada instrumento com seus respectivos dados psicométricos, a discussão e os resultados com as vantagens e limitações dos instrumentos listados.

Nesse sentido, foram considerados as características dos domínios investigados de cada instrumento, o tempo de aplicação, o ponto de corte, os escores ou percentis, a confiabilidade, a sensibilidade, a especificidade, além de suas respectivas referências, bem como as características qualitativas das vantagens e limitações de cada teste, escala ou bateria.

Para que isso fosse possível, foram pesquisados em bases eletrônicas artigos, dissertações e teses em português e inglês produzidos no Brasil e em outros países, com o intuito de selecionar trabalhos científicos que atendessem às exigências dos objetivos da pesquisa, e que possibilitassem o refinamento dos dados pesquisados. Alguns artigos mais antigos foram incorporados ao trabalho devido à relevância deles, por serem estudos clássicos sobre o tema. As buscas foram realizadas a partir das bases de dados BVS Psicologia (DeCS), LILACS, SCIELO, PsycINFO, PubMed, a partir dos seguintes descritores: Envelhecimento, Demências, Neuropsicologia e Rastreio Cognitivo.

\section{DESENVOLVIMENTO}

Segundo Malloy-Diniz et al. (2010) com o aumento da população idosa no Brasil, doenças que causam demência ${ }^{[2]}$ tornaram-se, ao lado dos transtornos depressivos, as enfermidades neuropsiquiátricas de maior prevalência na terceira idade. Antes do DSM-5 demência era definida como uma síndrome caracterizada pelo declínio progressivo e global de memória, associado ao déficit de uma ou mais funções cognitivas, como linguagem, apraxias, agnosia, Funções Executivas (FE), com uma 
intensidade que prejudicasse o desempenho social diário ou ocupacional da pessoa (APA, 2002).

Hoje, com os novos critérios do DSM-5, demência foi caracterizada como Transtorno Neurocognitivo Maior (TNM) devido aos subtipos a seguir: Doença de Alzheimer (DA), degeneração Lobar Frontotemporal (DLFT), Doença com Corpos de Lewy (DCL), Doença Vascular (DV), Lesão Cerebral Traumática (LCT), uso de substância/medicamento, Infecção por HIV, Doença do Príon (DPR), Doença de Parkinson (DPK) e Doença de Huntington (DH). Vide (QUADRO-1) para apresentação dos critérios diagnósticos comuns a todos os subtipos anteriormente listados.

As síndromes demenciais, classificam-se em duas categorias: degenerativas e não degenerativas. As demências não degenerativas são decorrentes de traumatismos, acidentes vasculares, processos infecciosos, tumores, deficiências nutricionais, dentre outras patologias.

QUADRO - 1. Critérios diagnósticos das demências - DSM-5

TRANSTORNO NEUROCOGNITIVO MAIOR

Critérios diagnósticos:

A. Evidências de declínio cognitivo importante a partir de nível anterior de desempenho em um ou mais domínios cognitivos (atenção complexa, função executiva, aprendizagem e memória, linguagem, perceptomotor ou cognição social),

com base

em:

I. Preocupação do indivíduo, de um informante com conhecimento ou do clínico de que há declínio significativo na função cognitiva; e II. Prejuízo substancial no desempenho cognitivo, de preferência documentado por teste neuropsicológico padronizado ou, em sua falta, por outra investigação clínica quantificada.

B. Os déficits cognitivos interferem na independência em atividades da vida

Disponível em: https://www.nucleodoconhecimento.com.br/psicologia/vantagens-elimitacoes 
diária (i.e., no mínimo, necessita de assistência em atividades instrumentais complexas da vida diária, tais como pagamento de contas ou controle medicamentoso).

C. Os déficits cognitivos não ocorrem exclusivamente no contexto de delirium.

D. Os déficits cognitivos não são mais bem explicados por outro transtorno mental (p. ex., transtorno depressivo maior, esquizofrenia).

Fonte: American Psychiatric Association - (APA, 2014).

Já as demências degenerativas como a DA, têm sua origem predominantemente no córtex cerebral; e outras, como a doença de Huntington, têm origem subcortical. Tal divisão entre demência cortical e subcortical consiste na localização da lesão (ARAÚJO; NICOLI, 2010).

Os tipos mais comuns de demência em idosos são a DA e a Demência Vascular (DV), sendo que o subtipo mais comum de DV é a Demência Vascular Isquêmica Subcortical (DVIS), a qual tem início lento e curso clínico gradual caracterizado por disfunção executiva e perda leve de memória. Os dois tipos de demências, a DVIS e a DA caracterizam-se pelo início insidioso e curso progressivo (MATIOLI, 2005; MATIOLI; CARAMELLI, 2010).

O diagnóstico etiológico da maioria das síndromes demenciais é baseado em exames laboratoriais, de neuroimagem e do perfil neuropsicológico característico. Tais aspectos são importantes para o diagnóstico diferencial das demências (ARAÚJO; NICOLI, 2010). Já o diagnóstico definitivo depende do exame neuropatológico e uma avaliação clínica cuidadosa (NETO; TAMELINI; FORLENZA, 2005). A avaliação cognitiva atua como importante auxílio diagnóstico, uma vez que há dificuldades de identificar os quadros demenciais precocemente apenas por meio de avaliação clínica e exames médicos de rotina. Portanto, tornam-se necessários utilizar tanto os instrumentos de rastreio cognitivo usados no início do processo 
diagnóstico quanto os demais instrumentos neuropsicológicos utilizados nas investigações mais amplas (MALLOY-DINIZ et al., 2010).

A função do instrumento de rastreio é distinta da AN. O rastreio aponta para a possibilidade ou não de demência, e a AN investiga mais detalhadamente e especificamente os déficits apontados pelo rastreio cognitivo, confirmando ou não a suspeita de demência. A AN tem sua importância quando o quadro clínico é ambíguo ou complexo, propiciando uma diferenciação precoce e confiável entre os diferentes tipos de demência, entre demência e envelhecimento normal, ou entre demência e outras afecções (MALLOY-DINIZ et al., 2010).

Conforme Aprahamian et al. (2008), são exemplos de testes utilizados para a avaliação inicial de pacientes com suspeita de comprometimento cognitivo: o Miniexame do Estado Mental (MEEM), o teste do Desenho do Relógio (TDR), o teste de FV para categorias. Além de testes mais simples como os mencionados para triagem inicial e avaliação mais breve, há baterias neuropsicométricas mais abrangentes como o Consortium to Establish a Registry for Alzheimer's Disease (CERAD), a Mattis Dementia Rating Scale e o CAMDEX, traduzido para o português.

Malloy-Diniz et al. (2010) aponta, além dos instrumentos citados pelo autor acima, o Teste Breve de Performance Cognitiva (SKT), a Bateria de Avaliação Frontal (FAB) e o Exame Cognitivo de Addenbrooke-revisado (ACER). Neste sentido, sabe-se que os instrumentos de rastreio cognitivo breves são largamente utilizados para detecção de déficits que podem estar associados a processos demenciais (MALLOY-DINIZ et al., 2010; APRAHAMIAN et al., 2008).

\section{INSTRUMENTOS DE RASTREIO COGNITIVO}

A seguir serão apresentados de forma sucinta e objetiva, os instrumentos de rastreio cognitivo analisados neste trabalho com suas respectivas características. 


\section{MINI EXAME DO ESTADO MENTAL (MEEM)}

O MEEM é um teste de rastreio cognitivo global que foi desenvolvido por Folstein et al. (1975) e seu objetivo inicial foi de ser uma avaliação clínica prática de mudança do estado cognitivo em pacientes geriátricos (CHAVES et al., 2011). No Brasil foi traduzido e apresentado por Bertolucci et al. (1994). O teste possui uma pontuação máxima de 30 pontos, e seu ponto de corte varia de acordo com a escolaridade do paciente (MONTIEL et al., 2014). O teste examina orientação espacial e temporal, memória de curto prazo (imediata / atenção) e evocação, habilidades de linguagem, visuo-espaciais, cálculo e praxia. A pontuação varia de 0 a 30 , sendo que o escore total diminui à medida que o comprometimento cognitivo aumenta. Sua aplicação não demanda mais do que cinco minutos e utiliza-se apenas um lápis e uma folha de papel (MALLOY-DINIZ et al., 2010).

\section{TESTE DO DESENHO DO RELÓGIO (TDR)}

O TDR é amplamente utilizado como teste de rastreio cognitivo, de simples aplicação e de rápida execução, que avalia diversas dimensões cognitivas, como memória, função motora, função executiva e compreensão verbal (APRAHAMIAN et al., 2008). O teste foi traduzido e adaptado para a língua portuguesa por Bottino e colaboradores (1999), além de ser estudado no Brasil por Martinelli, Aprahamian e Cachioni (2004); Aprahamian et. al. (2008) e Atalaia-Silva e Lourenço (2008), entre outros. O TDR prioriza as funções visuo-espaciais, bem como a capacidade executiva de reconstruir a partir de um comando verbal, a memória de um relógio em uma imagem gráfica. Essa praxis construtiva envolve análise visuoperceptual, execução motora, atenção, compreensão da linguagem e conhecimento numérico. $O$ TDR pode ser aplicado de formas variadas, pois cada autor estabeleceu seu próprio critério em relação às instruções e à correção (MALLOY-DINIZ et al., 2010). O tempo médio de aplicação do TDR é de, aproximadamente, 5 minutos. Utiliza-se apenas uma folha de papel e um lápis (ATALAIA-SILVA; LOURENÇO, 2008). 


\section{FLUÊNCIA VERBAL (FV)}

O Teste de FV é uma tarefa sensível para avaliar as funções do lobo frontal, especialmente área pré-frontal esquerda. É extremamente simples e avalia a memória semântica (conhecimento geral sobre o mundo, fatos, palavras, sem relação com o momento do seu aprendizado). Existem dois tipos de tarefas de fluência verbal: semântica e fonêmica. No teste de fluência semântica o sujeito tem que dizer tantas palavras quanto possível que pertença a uma determinada categoria (por exemplo, animais, frutas), com normas para idosos em Brucki e Rocha (2004), Machado et al., (2009), Silva et al. (2010). Já a tarefa de fluência fonêmica exige que a pessoa fale tantas palavras quanto possível que inicie, geralmente, com a letra ( $F)$, (A) e (S), uma de cada vez por 1 (um) minuto cronometrado, com normas em Machado e et al., (2009). A pontuação varia de acordo com a produção de palavras pela pessoa avaliada, onde cada palavra correta é pontuada com 1 ponto, obtendo-se o total. A análise é feita comparando o total de palavras com a média e desvio padrão esperado para cada faixa etária e o grau de escolaridade, sendo que a maior pontuação indica o melhor desempenho na realização do teste. Os testes requerem para sua aplicação, cronômetro, folha para registro das respostas ou gravador (MACHADO et al., 2009; BRUCKI et al., 1997; CAIXETA; FERREIRA, 2012).

\section{BATERIA DE AVALIAÇÃO FRONTAL (FAB)}

A FAB é um instrumento de triagem ou rastreio neurocognitivo, criado por Dubois et al. (2000) destinado à avaliação das FE frontais [a DA apresenta déficits importantes nas funções executivas], para fins de identificação e caracterização da síndrome disexecutiva, tanto em seus componentes executivos quanto motores, em um único instrumento (MOURA, 2008). Trata-se de um instrumento simples e de aplicação rápida, cerca de 10 minutos, com boa validade para exame das FE em população idosa. A versão brasileira foi proposta por Beato et al. (2007) que traduziram os seis subtestes da versão original. Os subtestes avaliam a formação de conceitos 
(abstração); FV (flexibilidade mental); programação motora; suscetibilidade à interferência (tendência à distração); controle inibitório e autonomia. Também analisaram a influência da idade e da escolaridade em cada componente. A pontuação varia de 0 a 18, sendo que a maior pontuação indica o melhor desempenho na realização do teste (BEATO et al. 2007; MALLOY-DINIZ et al., 2010, 2013).

\section{TESTE BREVE DE PERFORMANCE COGNITIVA (SYNDROM KURZTEST, SKT)}

Desenvolvido por Erzigkeit (1989) o SKT é um teste de rastreio cognitivo de aplicação rápida, 10 a 15 minutos, capaz de detectar déficits que caracterizam os estágios iniciais de demência ao avaliar as funções amnésticas e atencionais, com base na velocidade de processamento das informações. Pode ser utilizado em pacientes em leitos hospitalares. É composto por nove sub testes. Os itens I, III, IV, V, VI e VII medem a velocidade de processamento da atenção; já os sub testes II, VIII e IX avaliam a evocação da memória imediata, tardia e de reconhecimento. O tempo é cronometrado para cada sub teste, sendo este de 60 segundos. O escore total, que pode variar de 0 a 27 pontos, fornece dados sobre a severidade da doença, sendo que, quanto mais alto o resultado, mais importante é o prejuízo cognitivo. Resultados também podem ser avaliados quanto aos domínios de memória e atenção, permitindo que se dimensione a homogeneidade e a discrepância destes (FLAKS, 2008).

\section{ESCALA DE AVALIAÇÃO DE EDMÊNCIA (DRS)}

A Dementia Rating Scale (DRS) desenvolvida por Steven Mattis (1988) é usada na avaliação do estado cognitivo geral e é considerada por muitos pesquisadores como um instrumento muito útil para avaliar pacientes com demência e tem sido frequentemente utilizada tanto na prática clínica quanto na pesquisa. É fácil de aplicar, com duração de cerca de 30 a 40 minutos. As 36 tarefas são agrupadas em 
5 subescalas cada uma avalia diferentes áreas cognitivas e fornece uma pontuação parcial. As subescalas com suas respectivas pontuações parciais são: Atenção, 37 pontos; Iniciação/Perseveração (I / P), 37 pontos; Construção, 06 pontos; Conceitualização, 39 pontos; e Memória, 25 pontos. O total possível é de 144 pontos, sendo que a maior pontuação indica o melhor desempenho na realização do teste (MATTIS, 1988).

\section{AVALIAÇÃO COGNITIVA MONTREAL (MOCA)}

O MoCA é um instrumento de rastreamento cognitivo breve criado por Nasreddine et al. (2005) para detectar Comprometimento Cognitivo Leve (CCL). Trata-se de um teste de uma página, traduzido e adaptado para 30 línguas, ou seja, vinte e sete países traduziram suas versões (MEMÓRIA, 2013). O teste avalia diversas funções cognitivas como as FE, habilidades visuo-espaciais, nomeação, dígitos, sentença, recuperação da memória, raciocínio abstrato e orientação, contribuindo com o diagnóstico do CCL e de demência. O tempo de aplicação é de aproximadamente 20 minutos e a pontuação máxima possível é de 30 pontos. Para CCL o ponto de corte é de 26 pontos, onde acima disso é considerada normal (CECATO, 2014).

\section{CONSORTIUM TO ESTABLISH A REGISTRY FOR ALZHEIMER'S DISEASE (CERAD)}

O Consórcio para Estabelecer um Registro para a DA - Bateria CERAD, foi desenvolvida por Moms et. al. (1989). No Brasil foi adaptada por Bertolucci et al. (2001) a qual avalia as funções cognitivas utilizando os sub testes de Fluência Verbal, Teste de Nomeação de Boston (versão reduzida), Memória da Lista de Palavras, Praxia Construtiva, Evocação Tardia da Lista de Palavras, Reconhecimento da Lista de Palavras, Evocação de Praxia e o MEEM. Uma nota de corte é indicada para cada subdomínio. O protocolo completo da CERAD inclui ainda uma entrevista sobre o declínio cognitivo, doença vascular cerebral, doença de Parkinson, depressão, uso de drogas que afetam a cognição, abuso de álcool e 
complicações físicas e neurológicas. Os sub testes são aplicados de 20 a 30 minutos, com isso facilitando o uso com idosos (MOMS et. al., 1989; BERTOLUCCI et al., 2001; RIBEIRO et al., 2010; FILLENBAUM et al., 2011; MALLOY-DINIZ et al., 2013). A pontuação varia de acordo com cada subteste da bateria, sendo que as maiores pontuações indicam o melhor desempenho na realização dos sub testes e consequentemente da bateria.

\section{CAMBRIDGE COGNITIVE EXAMINATION-REVISED (BR-CAMCOG-R)}

O Br-CAMCOG-R é um instrumento que mede a função cognitiva global e corresponde a versão brasileira do Cognitive Examination-Revised (CAMCOG). Foi desenvolvido, validado e adaptado por Paradela; Lopes e Lourenço (2009 a;b), a partir da Cambridge Cognitive Examination-Revised (CAMCOG-R), que é a secção B da versão revista do Exame Cambridge para Transtornos Mentais do Idoso (CAMDEX-R), desenvolvido na Universidade de Cambridge, Inglaterra. $\mathrm{O} \mathrm{Br}$ CAMCOG-R incorporou alguns instrumentos de triagem para distúrbios cognitivos amplamente utilizados na investigação epidemiológica, como o MEEM e 8 dos 10 itens do teste Hodgkinson Abbreviated Mental (AMT). O CAMCOG-R é subdividido em 8 subescalas, quais sejam: orientação (hora e local), a linguagem (compreensão e expressão), memória (incidental, remota e recente, nova aprendizagem), atenção, cálculo, práxis, percepção e FE (pensamento abstrato, fluência ideativo e raciocínio visual). O instrumento é aplicado em média em 43 minutos. Quanto maior a pontuação total, melhor o desempenho no teste (PARADELA; LOPES E LOURENÇO, 2009 a; 2009 b).

\section{ADDENBROOKE'S COGNITIVE EXAMINATION (ACE-R - VERSÃO REVISADA)}

De acordo com Noone (2015) a precisão diagnóstica da ACE como um instrumento de rastreio cognitivo breve, levou à sua adoção generalizada. O Exame Cognitivo original de Addenbrooke (ACE) foi desenvolvido no Medical Research Cognition and

Disponível em: https://www.nucleodoconhecimento.com.br/psicologia/vantagens-elimitacoes 
Brain Sciences Unit, em Cambridge, Inglaterra, no final de 1990 como uma bateria de testes simples para beira de leitos projetado para detectar demência leve e diferenciar a DA de Demência Frontotemporal. A ACE original incluía o MEEM ou teste de Folstein (1975), juntamente com itens fronto-executivos e visuo-espaciais.

No entanto, surgiram possibilidades de melhoria no ACE, o que levou ao desenvolvimento de Cognitive Revised-Exame do Addenbrooke (ACE-R) para facilitar o uso transcultural e melhorar a sensibilidade. Os 26 componentes originais foram combinados para produzir cinco subscores, cada uma representando um domínio cognitivo específico: atenção / orientação (18 pontos), memória (26 pontos), fluência (14 pontos), línguagem (26 pontos) e função visuo-espacial (16 pontos), perfazendo o total de 100 pontos. Uma nota de corte é indicada para os cinco subdomínios e requer entre 12 e 20 minutos (média de 16 minutos) para ser aplicada, onde na apuração quanto maior a pontuação melhor o desempenho na bateria. A sensibilidade ACE-R para demência leve é de 84\% a 94\%, dependendo do ponto de corte Noone (2015). No Brasil a ACE-R foi traduzida e adaptada para a nossa população por Carvalho e Caramelli (2007) e a adaptação transcultural foi feita por Carvalho (2009).

\section{DISEASE ASSESSMENT SCALE (ADAS-COG)}

ADAS-Cog é uma das escalas mais utilizadas em ensaios clínicos com medicação que avalia memória, linguagem, praxias e compreensão de ordens. Ela foi adaptada para a população brasileira por Shultz, Siviero e Bertolucci (2001) a qual possibilita avaliação de domínios comumente prejudicados em quadros demenciais e distribuídos nas seções de Memória (50\%), Linguagem (28\%), Praxias (14\%) e Compreensão de ordens $(8 \%)$, sendo considerado na escala que quanto maior a pontuação pior o desempenho, e requer aproximadamente 30 a 45 minutos para ser administrada de acordo com o grau de comprometimento cognitivo (SHULTZ; SIVIERO; BERTOLUCCI, 2001; MALLOY-DINIZ et al., 2013). 


\section{RESULTADOS E DISCUSSÃO}

Abaixo serão apresentados os dados psicométricos de cada instrumento de rastreio cognitivo disponibilizado no (QUADRO-2).

Segundo Chaves et al. (2011) o MEEM é um instrumento de rastreio cognitivo que apresenta muitas vantagens em sua utilização, pois é de rápida aplicação, abarca todas as principais funções, apresenta alta especificidade e sensibilidade para identificar déficits cognitivos e pode ser usado por diversos profissionais de saúde; com isso, o MEEM tem sido efetivamente o instrumento mais usado e citado em estudos brasileiros para declínio cognitivo de idosos, inclusive para avaliação cognitiva de beira de leito, "sugerindo ampla utilização e parâmetro para comparação com outros testes" (MALLOY-DINIZ et al., 2013).

Quanto às limitações do MEEM, existe uma grande heterogeneidade entre a escolarização entre populações e mesmo entre regiões de um mesmo país e o MEEM é fortemente influenciado pela educação. Neste sentido, tem-se observado que o nível educacional tem sido frequentemente avaliado usando-se o nível de escolaridade, o que pode não ser uma boa medida direta de nível educacional, pois existe uma grande heterogeneidade nos padrões de escolaridade entre populações e em regiões do nosso país (BRUCKI, 2011). A função executiva não é tão bem avaliada no MEEM embora esteja presente inicialmente em diversos casos de síndrome demencial como manifestação quase que exclusiva (APRAHAMIAN, 2008). Além disso, o MEEM identifica corretamente casos de DA leve, mas não apresenta boa acurácia para diferenciar os diversos subtipos de CCL (DINIZ et al., 2008; DA MOTA, 2008).

Uma pesquisa com idosos no Brasil por meio de revisão sistemática, Melo e Barbosa (2015) descobriram que: apesar da ampla utilização do MEEM no Brasil, faltam padronização e evidências de validade para essa medida. 
É de fundamental importância salientar que como qualquer exame do desempenho cognitivo o MEEM não deve ser usado de forma isolada (FOLSTEIN; FOLSTEIN; MCHUGH, 1975).

O TDR parece ser um bom teste de rastreio cognitivo para demência. Na versão de Sunderland e Colaboradores (1989) faz parte da bateria CAMDEX-R (BOTTINO et al., 1999; APRAHAMIAN; MARTINELLI; NERI; YASSUDA, 2009). Esse instrumento é um teste de rastreio cognitivo breve muito utilizado ao qual, Freitas e Simões (2010) afirmam que a simplicidade, a facilidade de administração, o reduzido tempo de aplicação, a instruções de fácil compreensão, a aceitação por parte dos pacientes, a ampla gama de funções cognitivas avaliadas como memória, função motora, função executiva e compreensão verbal; e o fato de proporcionar um registro visual / gráfico objetivo, e de ser um instrumento adequado no exame de idosos, constituem algumas das vantagens que têm sido apontadas ao teste. Além disso, o poder discriminativo do TDR entre a população com demência e a população normal tem-se revelado robusto em vários estudos (FREITAS, 2010).

Shulman (2000) observou em um estudo de revisão, que a maioria dos estudos do TDR apresentou medidas de sensibilidade e especificidade em torno de $85 \%$ e grande aceitabilidade de execução pelos pacientes (ATALAIA-SILVA, 2008). Como instrumento único de rastreio o TDR apresenta limitações. A principal limitação do teste aponta para a variabilidade dos protocolos de administração e cotação, geradora de divergências que dificultam a comparação dos resultados entre vários estudos. Outro fator não menos importante é a escassez de estudos normativos que descrevam o desempenho dos sujeitos cognitivamente normais (FREITAS; SIMÕES, 2010). Além disso, o TDR exige diversas funções cognitivas, tanto motoras quanto perceptivas, para a sua conclusão bem-sucedida (SILVA et al., 2015).

Aprahamian et al. (2008) fizeram um estudo onde avaliaram 62 pacientes idosos com baixa escolaridade e constataram que o TDR não é um bom teste de rastreio para DA em estágio inicial com baixa escolaridade (menos de 4 anos). Atalaia-Silva 
e Lourenço (2008) também acharam acurácia reduzida para população de baixa escolaridade. O TDR parece revelar menor sensibilidade na deteç̧ão das diferenças entre os vários tipos de demência. Nesse sentido, é prudente utilizá-lo integrado a uma bateria de rastreio cognitivo ou em combinação com outros instrumentos, como o MEEM ou o SKT (FREITAS; SIMÕES, 2010).

O FV avalia a memória semântica e fonológica. É um teste extremamente simples, de fácil aplicação e tempo reduzido (MACHADO et al., 2009; BRUCKI et al., 1997; CAIXETA; FERREIRA, 2012). Ele também pode ser considerado um indicador das FE, pois envolve a capacidade de busca e recuperação de dados armazenados na memória de longo prazo. A tarefa de FV exige habilidades de organização, autorregulação e memória operacional. Contudo, existem algumas limitações a serem consideradas: o desempenho em FV deve ser interpretado à luz das informações sociodemográficas e as variáveis do teste são influenciadas por sexo, idade e escolaridade, com melhor desempenho a favor dos homens (SILVA et al., 2010). Além disso, na DA ocorre comprometimento acentuado de memória episódica e também maior comprometimento semântico-lexical. Ademais, o comprometimento de memória episódica é central para muitos quadros demenciais e essa função não é avaliada pelo FV, constituindo em outro limite do teste (MATIOLI, 2005).

A FAB, além de ser um instrumento simples, de aplicação rápida, com boa validade para exame das FE em população idosa Malloy-Diniz et al. (2013), fornece uma medida objetiva para distinguir DFT da DA em pacientes levemente dementes com uma sensibilidade de $77 \%$ e especificidade de $87 \%$ (SLACHEVSKY et al., 2004). Também tem sido largamente utilizada em diversos grupos de pacientes como doença de Parkinson, doença de Huntington entre outras condições (BEATO, 2012).

Quanto à limitação da bateria, em estudo realizado por Beato, Nitrini, Formigoni, Caramelli (2007) para avaliar o desempenho de indivíduos brasileiros saudáveis, correlacionado com gênero, idade, educação e escores do MEEM, a versão 
brasileira da FAB sofreu influência do nível de escolaridade, mas não da idade e do gênero.

Flaks (2008) fez um estudo de validação e propriedades diagnósticas do SKT no qual concluiu que os achados sugerem que o teste é um instrumento adequado de rastreio cognitivo para diferenciar a conversão entre CCL e DA. Contudo, o teste é levemente influenciado pela escolaridade e pode não discriminar adequadamente sujeitos com CCL de idosos normais.

A DRS, ou escala Mattis, possui vantagens por detalhar domínios prejudicados ou preservados, além de apresentar boa sensibilidade para formas mais severas de DA. Também tem sido utilizada para a detecção precoce de demência e diagnóstico diferencial entre pacientes com DA de intensidade leve e indivíduos controles, pacientes com CCL de controles, e pacientes com CCL de pacientes com DA de intensidade leve; e também é considerado um bom instrumento de diagnóstico diferencial entre DA e outras demências como a vascular (PORTO et al., 2003; MALLOY-DINIZ et al., 2013; PORTO, 2006; FOSS et al., 2013). Em comparação com outras baterias breves, a DRS apresenta algumas vantagens, pois fornece informações mais detalhadas sobre as funções cognitivas que são prejudicadas ou preservadas, uma vez que realiza uma avaliação mais aprofundada de um maior número de áreas cognitivas, bem como maior sensibilidade a formas mais graves de doença de DA. O valor do DRS tem sido reafirmado pelo fato de um número crescente de estudos ter mencionado o uso desta escala no diagnóstico e discriminação de doentes com DA daqueles com outras formas de demência, tais como doença de Parkinson, Doença de Huntington e Demência Vascular (PORTO et al., 2003; FOSS et al., 2013).

Em um estudo realizado por Porto et al. (2003) para verificar a acurácia diagnóstica da versão brasileira da DRS, com análise das variáveis idade, escolaridade e nota de corte 122, apurou-se sensibilidade de $91,7 \%$ e especificidade de $87,8 \%$, a qual demonstrou boa acurácia diagnóstica na discriminação entre pacientes com DA leve

RC: 22961

Disponível em: https://www.nucleodoconhecimento.com.br/psicologia/vantagens-elimitacoes 
e indivíduos controles. Com relação à limitação do teste, um estudo de Foss et al. (2005) já havia apontado que o analfabetismo é um fator determinante no rebaixamento nos escores da DRS, podendo gerar erros diagnósticos. Escolaridade, idade e fatores culturais também interferem na precisão do teste (FOSS et al., 2005).

Quanto ao MoCA, no estudo original Nasreddine et al. (2005) utilizou uma amostra de 277 idosos e propuseram uma pontuação de corte de 26 pontos para detectar 90\% dos indivíduos com CCL e 100\% dos indivíduos com DA. Em contrapartida, a sensibilidade para detectar CCL usando o MEEM foi de apenas $18 \%$ usando a mesma pontuação de corte de 26 pontos. Enquanto o MEEM apresentou uma sensibilidade de $78 \%$ para discriminar pacientes com DA leve (com valores de corte habituais), a sensibilidade do MoCA foi de $100 \%$.

No Brasil, Memória et al. (2013) desenvolveram o estudo de validação do MoCA, encontrando boas características psicométricas para o instrumento. Em estudo realizado por Cecato et al. (2014) o MoCA mostrou ser o teste com maior valor preditivo para diferenciar DA de CCL e também diferenciar CCL dos controles normais. A sensibilidade e a especificidade encontradas foram, respectivamente, $82,2 \%$ e $92,3 \%$. Além disso, o teste demonstrou correlação significativa com a variável idade e os testes MEEM, CAMCOG, TDR, de FV e Questionário de Atividades Funcionais de Pfeffer (QAFP), instrumentos já validados e amplamente utilizados no Brasil.

O MoCA, além de ser um instrumento de triagem breve e avaliar uma gama de funções cognitivas foi o melhor para diferenciar DA dos casos de CCL (BERTOLUCCl et al., 2001). Roalf et al. (2012) indicam que é uma ferramenta de avaliação global superior ao MEEM, particularmente nos estágios iniciais de declínio cognitivo. Assim como outros instrumentos de rastreio cognitivo o MoCA também têm suas limitações: indivíduos analfabetos têm dificuldades de executar as tarefas (SARMENTO, 2009).

RC: 22961

Disponível em: https://www.nucleodoconhecimento.com.br/psicologia/vantagens-e$\underline{\text { limitacoes }}$ 
Com relação às vantagens da bateria CERAD, estas são relacionadas à sua amplitude ao ser comparada a outros testes de rastreio, e ainda, permite a confrontação de dados entre grupos de pesquisa, além de ser resumida em 30 minutos, facilitando o seu uso em idosos (BERTOLUCCl et al., 2001). Já em relação às limitações da CERAD, Ribeiro et al. (2010) fizeram um estudo avaliando 158 idosos, no qual os resultados mostraram diferença dependente de anos de escolaridade, gênero, estado conjugal e saúde percebida. Em todos os subtestes escores mais elevados foram associados à escolaridade mais alta, com exceção do teste de reconhecimento.

Apesar de a CERAD ser considerada útil para o auxílio em diagnóstico diferencial de demência e funcionamento normal em idosos, um cuidado deve ser observado no uso isolado de subtestes da bateria para a avaliação (MALLOY-DINIZ et al., 2013).

Antes do advento da 5ª edição do DSM, uma das vantagens do CERAD era ter sido construído a partir de critérios diagnósticos bem estabelecidos, formulados pelo DSM-IV (APA, 2002) e Classificação internacional de doenças e problemas relacionados à saúde CID-10 (OMS, 1998). Estes critérios focavam o déficit de memória como central e imprescindível para o diagnóstico, além de mais outra área cognitiva comprometida. Inclusive, tais critérios foram adotados para o diagnóstico de DA pelo National Institute of Neurological Disorders and Stroke - Alzheimer Disease and Related Disorders (NINCDS-ADRDA) (MCKHANN et al., 1984; 2011) com uma sensibilidade de $80 \%$ para o diagnóstico de DA provável, o que levou a criação do CERAD em 1986 (BERTOLUCCI, 2001).

Atualmente, os critérios sugeridos pelo DSM-5 (APA, 2014) mudaram e, em parte, a utilização da CERAD pode apresentar algumas limitações. Pois, para os critérios atuais não é mais preciso, necessariamente, ter déficit de memória como principal critério diagnóstico de demência.

O Br-CAMCOG-R tem a vantagem de oferecer uma medida resumo da função cognitiva global, permitindo mudanças da nota de corte de acordo com a idade e 
escolaridade. O teste tem um excelente nível de estabilidade com o tempo, tanto para o conjunto de itens no teste e para a maioria dos itens das subescavas. O Coeficiente de Correlação Intraclasse $(\mathrm{CCl})$ para o conjunto de itens no $\mathrm{Br}$ CAMCOG-R foi alto, 0,93 $(0,89-0,95)$ e os escores CCI para o conjunto de itens do teste, bem como para os itens das subescalas separadamente e os itens MEEM foram excelentes $(>0,92)$, com intervalos de confiabilidade estreitos. Portanto o teste pode ser útil em estudos epidemiológicos e em clínicas especializadas na avaliação das funções cognitivas dos idosos (PARADELA; LOPES; LOURENÇO, 2009 a; b). Outra vantagem é a ausência de diferenças significativas nos índices de correlação para gênero, idade e anos de estudo (MALLOY-DINIZ et al., 2013).

Com relação à limitação da Br-CAMCOG-R, Paradela e Lourenço (2014) fizeram um estudo no qual avaliaram 189 idosos de baixa escolaridade e analfabetos, com idade média de 77 anos e nível médio de escolaridade de 3 anos, sendo $56(29,6 \%)$ participantes com demência, para determinar a acurácia da versão brasileira do $\mathrm{Br}$ CAMCOG-R. Eles encontraram que o teste foi útil para identificar casos de demência em idosos com níveis de alfabetização médios e baixo, mas inadequado para os analfabetos.

Carvalho (2009) fez o estudo de adaptação transcultural da ACE-R (Revisada), avaliando 31 pacientes com DA provável leve e 114 idosos cognitivamente saudáveis, objetivando investigar o desempenho de ambos os grupos. Com esse estudo apuraram que a ACE-R é um instrumento de boa acurácia e de propriedades diagnósticas satisfatórias para a avaliação cognitiva em nosso meio, sendo uma bateria de testes simples com elevada sensibilidade e especificidade (100\% e 82,3\% respectivamente) para detectar demência em estágio leve. Ela é particularmente útil para diferenciar a DA de demência frontotemporal, além de avaliar com eficiência vários domínios cognitivos, comumente afetados em estágios iniciais da doença. Por outro lado, há que ressaltar algumas limitações de sua aplicação. A escolaridade influenciou em todos os escores. Já a idade interferiu nas subescalas Memória, Fluência e pontuação total (CARVALHO; CARAMELLI, 2007; CARVALHO, 2009). 
A ADAS-Cog não é um instrumento específico para o diagnóstico, mas seus escores são capazes de diferenciar indivíduos de grupos clínicos com DA e de grupos sem demência (MALLOY-DINIZ et al., 2013).

Chu et al. (2011) realizaram um estudo para verificar a confiabilidade e validade de uma versão reduzida da ADAS-Cog na avaliação da DA entre chineses idosos, em Hong Kong, na qual obtiveram os seguintes resultados: sensibilidade de $90 \%$, especificidade de $94,7 \%$ e precisão global de $92,3 \%$.

Com relação à suas limitações a ADAS-Cog é um instrumento útil para identificar demência leve, embora possa haver uma sobreposição quando comparados indivíduos dementes com alta escolaridade de indivíduos não dementes com baixa escolaridade. A educação é variável, o que pode ser um grande problema, pois influencia o desempenho em avaliação cognitiva (SCHULTZ; SIVIERO; BERTOLUCCI, 2001; MALLOY-DINIZ et al., 2013). Não foram encontrados estudos brasileiros de confiabilidade, sensibilidade e fidedignidade do instrumento. 
QUADRO - 2 - Instrumentos de rastreio cognitivo com suas respectivas características psicométricas.

\begin{tabular}{|c|c|c|c|c|c|c|c|}
\hline \multirow{2}{*}{$\begin{array}{l}\text { INSTRUMEN- } \\
\text { TOS }\end{array}$} & \multirow{2}{*}{$\begin{array}{l}\text { TEMPO DE } \\
\text { APLICA- } \\
\text { CAOA }\end{array}$} & \multirow{2}{*}{$\begin{array}{l}\text { DOMINIOS } \\
\text { INVESTIGADOS }\end{array}$} & \multicolumn{4}{|c|}{ NORMAS } & \multirow[b]{2}{*}{ REFERËNCIAS } \\
\hline & & & $\begin{array}{l}\text { Ponto de Corte / Escores I } \\
\text { Percentis }\end{array}$ & $\begin{array}{l}\text { Confiabilidade } \\
\text { Teste-reteste / CCl }\end{array}$ & Sensibilidade & Especificidade & \\
\hline $\begin{array}{l}\text { Mini-Exame do } \\
\text { Estado Mental } \\
\text { (MEEM) }\end{array}$ & 5 min. ${ }^{\circ}$ & $\begin{array}{l}\text { Orientacão espacial e } \\
\text { temporal, memória de curto } \\
\text { prazo (imediata ou atencãa) } \\
\text { e evocacáa, habilidades de } \\
\text { linguagem, visuo-espaciais, } \\
\text { cálculo e praxia. }\end{array}$ & $\begin{array}{l}\text { Analfabetos: } \\
\text { Corte: } 13^{\circ} \\
\text { Baixa e Média Escolar.: } \\
\text { Corte: } 18^{\circ} \\
\text { Alta Escolaridade: } \\
\text { Corte: } 26^{\circ}\end{array}$ & $\begin{array}{l}\text { Coeficiente kappa: } 0,79^{\circ} \\
\text { Alfa de Cronbach: } 0,71^{\circ} \\
\text { Alfa de Cronbach: } 0,80^{\circ}\end{array}$ & $\begin{array}{l}\text { Analfabetos } \\
82,4 \%{ }^{\circ} \\
\text { Baixa/Média } \\
\text { Escolaridade } \\
75,6 \% \%^{\circ} \\
\text { Alta Escolaridade } \\
80,0 \%{ }^{\circ}\end{array}$ & $\begin{array}{l}\text { Analfabetos } \\
97,5 \% \%^{2} \\
\text { Baixa/Média } \\
\text { Escolaridade } \\
96,6 \% \text { a } \\
\text { Alta } \\
\text { Escolaridade } \\
95,6 \% \text { a } \\
\end{array}$ & $\begin{array}{l}{ }^{A} \text { Bertolucci et al. (1994); } \\
\text { L Lourenço et al. (2008); } \\
\text { CSantos et al. (2010); } \\
\text { 'Malloy-Diniz et al. (2010). }\end{array}$ \\
\hline $\begin{array}{l}\text { Teste do } \\
\text { Desenho do } \\
\text { Relógio (TDR) }\end{array}$ & $\begin{array}{l}5 \text { min. } \\
\text { (em média) }\end{array}$ & $\begin{array}{l}\text { Habilidades visuo-espaciais } \\
\text { (Percepcão visual), } \\
\text { habilidades construtivas ( } \\
\text { Praxia de construcâo), } \\
\text { funcôes executivas e } \\
\text { heminegligência }\end{array}$ & $\begin{array}{l}\text { Mendez: Corte } \leq 18^{a} \\
\text { Escolaridade: até } 4 \text { anos }>8 \\
\text { anos. } \\
\text { Shulman: Corte } \leq 3^{\circ} \\
\text { Escolaridade: até } 4 \text { anos }>8 \\
\text { anos. }\end{array}$ & $\begin{array}{l}\text { Esc. de Shulman: } 0,865^{\circ} \\
\text { Escalla de Mendez: } 0,935^{\circ} \\
\text { Método Manos: } 0,944^{\circ} \\
\text { Alfa de Cronbach: } 0,773^{\circ}\end{array}$ & $\begin{array}{l}85 \%^{\mathrm{b}} \\
65 \%^{\mathrm{c}} \\
77,8 \%^{\mathrm{d}}\end{array}$ & $\begin{array}{l}85 \%^{\mathrm{b}} \\
65 \%^{\mathrm{a}} \\
72,3 \%^{\mathrm{d}}\end{array}$ & $\begin{array}{l}\text { AAprahamian et al. (2008) } \\
\text { EAtalaia-Silva; Lourenco (2008). } \\
\text { CLourenco et al. (2008); Malloy-Diniz, } \\
\text { (2013, p.254); } \\
\text { "Nitrini et al. (2007). }\end{array}$ \\
\hline $\begin{array}{l}\text { Fluência Verbal } \\
\text { Semântica } \\
\text { (animais) } \\
\text { Fonológica } \\
\text { F,A,S }\end{array}$ & $\begin{array}{l}1 \text { min. }{ }^{\circ} \\
\text { (por } \\
\text { categoria) }\end{array}$ & $\begin{array}{l}\text { Memória Semântica e } \\
\text { Fonológica }\end{array}$ & $\begin{array}{l}\text { Até } 8 \text { anos de escolaridade } \\
\text { Corte: } 9^{\circ} \text {. } \\
\text { Alta escolaridade } \\
\text { Corte: } 13^{\circ} \\
\text { Corte }<15^{\circ}\end{array}$ & $\begin{array}{l}\text { F. V. Semântica: } \\
\text { CCI: } 0,53^{\circ} \\
\text { Coef. Pearson: } 0,72^{\circ} \\
\text { F. V. Fonológica: } \\
\text { CCl:0.61 } \\
\text { Coef. Pearson: } 0,77^{\circ} \\
\end{array}$ & $\begin{array}{l}\text { Analfabetos: } 75 \% \text { " } \\
\text { Baixa esc. } 100 \% \text { " } \\
\text { Média esc.: } 87 \% \%^{\circ} \\
\text { Alta esc.: } 86^{\circ} \\
67 \%{ }^{\circ}\end{array}$ & $\begin{array}{l}\text { Analfabetos: } 79 \% \text { " } \\
\text { Baixa escol. }: 84 \% \text { a } \\
\text { Média escol. } 88 \% \%^{a} \\
\text { Alta escol. } 67 \% \%^{\circ} \\
83,6 \% \%^{\circ}\end{array}$ & $\begin{array}{l}\text { A Brucki et al. (1997); } \\
\text { B Nitrini et al. (2007); } \\
{ }^{\circ} \text { Batitsta et al (2013); } \\
\text { DMachado et al. (2009). }\end{array}$ \\
\hline $\begin{array}{l}\text { Bateria de } \\
\text { Avaliação } \\
\text { Frontal (FAB) }\end{array}$ & 10 min. $^{c}$ & $\begin{array}{l}\text { FUNC̄õES EXECUTIVAS: } \\
\text { Formação de conceitos } \\
\text { (abstracãao); fluência verbal } \\
\text { (flexibilidade mental); } \\
\text { programaçấo motora; } \\
\text { suscetibilidade à } \\
\text { interferencia } \\
\text { (tendência à distração); } \\
\text { controle inibitório; } \\
\text { autonomia. }\end{array}$ & $\begin{array}{l}\text { (9-caso / 10-não-caso) }^{\text {d }} \\
\text { Escolaridade: }^{\circ} \\
\text { (escore médio) } \\
\text { 1 a } 3 \text { anos: } 10,9 ; \\
4 \text { a } 7 \text { anos: } 12,8 ; \\
8 \text { a } 11 \text { anos:13,8; } \\
12 \text { ou mais: } 15,3\end{array}$ & $\begin{array}{l}\text { Alfa Cronbach:0,78 } \\
\text { Coeficiente kappa: } 0,87^{\text {a }}\end{array}$ & $\begin{array}{l}77 \%{ }^{\mathrm{b}} \\
72 \%^{\mathrm{d}}\end{array}$ & $\begin{array}{l}87 \%^{\mathrm{b}} \\
61 \%^{\mathrm{d}}\end{array}$ & $\begin{array}{l}\text { A Dubois et al. (2000); } \\
\text { " Slachevsky et al. (2004); } \\
\text { 'Beato et al. (2007); Beato et al. } \\
\text { (2012); } \\
\text { DDe Paula et al. (2013). }\end{array}$ \\
\hline SKT & 10 a $15 \mathrm{~min}$ & $\begin{array}{l}\text { Velocidade de } \\
\text { processamento da stençẫo, } \\
\text { Evocaçäo da meméria } \\
\text { imedista, trardia e } \\
\text { reconhecimento. }\end{array}$ & $\begin{array}{l}0 \text { a } 4 \text { - Sem prejuizo } \\
\text { cognitivo: } \\
5 \text { a } 8 \text { - Dedínio cognitivo } \\
\text { muito leve; } \\
\text { 9 a } 13 \text { - Dedinio } \\
\text { Cognitivo Leve; } \\
\text { 14 a } 18 \text { - Declínio Cognitivo } \\
\text { Modergdo }\end{array}$ & $\begin{array}{l}\text { Alfa de Cronbach: } 0,83 \text { a } \\
0,88\end{array}$ & $\begin{array}{l}\text { Escolaridade } \\
\text { média ( } 5 \mathrm{a} 9 \\
\text { anos) e alta } \\
\text { escolaridade ( }>10 \\
\text { anos) - } 80,5 \% \text {; } \\
\text { Até } 4 \text { anos de } \\
\text { escolaridade e } \\
\text { analfabetos- } \\
75.0 \%\end{array}$ & $\begin{array}{l}\text { Escolaridade média } \\
\text { (5 a } 9 \text { anos) e alta } \\
\text { escolaridade ( }>10) \text { - } \\
80,3 \% \\
\text { Até } 4 \text { anos de } \\
\text { escolaridade e } \\
\text { ansalfabetos - } \\
56.7 \%\end{array}$ & Flaks (2008). \\
\hline $\begin{array}{l}\text { Escals Mattis } \\
\text { (DRS) }\end{array}$ & $\begin{array}{l}30 \mathrm{a} 40^{*} \\
\min \end{array}$ & 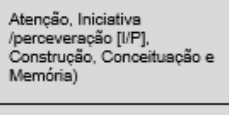 & $\begin{array}{l}122 / 123^{*} \\
<128^{\circ} \\
<123^{\circ} \\
<134^{\circ} \\
<124^{a} \\
\end{array}$ & $\begin{array}{l}\text { Coeficiente } \\
\text { Kapps: } 0.83^{2}\end{array}$ & $\begin{array}{l}91.7 \% " \\
90.0 \% " \\
78.2 \% " \\
73.3 \% " \\
93.3 \% " \\
\end{array}$ & $\begin{array}{l}87.8 \% " \\
89.3 \% " \\
76.8 \% " \\
72,7 \% " \\
91.7 \% " \\
\end{array}$ & 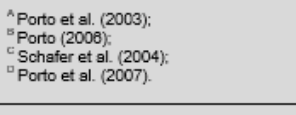 \\
\hline MoCA & $20 \mathrm{~min}^{.}$ & 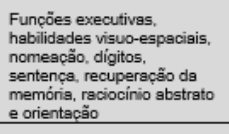 & $\begin{array}{l}\text { CCL corte: } 28^{\circ} \\
\text { CCL corte: } 28^{\circ}\end{array}$ & Alfa de Cronbach: $0,83^{\text {d }}$ & $\begin{array}{l}\text { CCL: } 84,09 \%^{*} \\
\text { CCL: } 82,2 \% \%^{\circ} \\
\text { CCL: } 900 \%^{\circ} \\
\text { DA: } 100 \% \%^{\circ}\end{array}$ & $\begin{array}{l}\text { CCL: } 60,0 \% \%^{\circ} \\
\text { CCL: } 92,3 \%^{\circ} \\
\text { CCL: } 87,0 \%\end{array}$ & $\begin{array}{l}\text { ^Davis et al. (2015): } \\
\text { "Decato et al. (2014): } \\
\text { " Nasreddine et al. (2005); } \\
{ }^{\circ} \text { Rahman et al. (2009). }\end{array}$ \\
\hline Bateria CERAD & 20 \& $30 \mathrm{~min}$. & $\begin{array}{l}\text { Memoria (fixação, evoçąão } \\
\text { ereconhec), lingugagem } \\
\text { (nom. e fuên. verbal). } \\
\text { Praxis (copia de desenhos } \\
\text { geometricos). Fun. } \\
\text { Executivgs (teste das } \\
\text { trilhss). MEEM. }\end{array}$ & $\begin{array}{l}\text { "Fluência: } 11 \\
\text { N. Boston: } 12 \\
\text { MEEM: } 28 \\
\text { Lista pslavras:13 } \\
\text { Praxia construtiva: } 9 \\
\text { Evocacaso de palavras: } 3 \\
\text { Reconhec. de Palavras: } 7 \\
\text { Evocacäo praxia: } 4\end{array}$ & $0,82 \times 1,0^{*}$ & $\begin{array}{l}\text { "Fluên. v. } 73.8 \% \\
\text { Nom. Bost: } 61,9 \% \\
\text { MEEM: } 97.6 \% \\
\text { Lista pal.: } 85,7 \% \\
\text { Prax Con.: } 81,0 \% \\
\text { Evoc. pal.: } 74,2 \% \\
\text { Rec. pal.: } 78.2 \% \\
\text { Evoc. Prax.: } 87,1 \% \\
\end{array}$ & $\begin{array}{l}\text { "Fluên. v. } 87,1 \% \\
\text { Nom. Bost: } 69,446 \\
\text { MEEM. } 75.3 \% \\
\text { Lista psl:: } 87,196 \\
\text { Prax Con.: } 51,8 \% \\
\text { Evoc. pal.: } 82,4 \% \\
\text { Rec. pal.: } 87,1 \% \\
\text { Evoc. prax.: } 87.1 \% \\
\end{array}$ & $\begin{array}{l}\text { AMoms et al. (1989): Eilleabsum et al. } \\
\text { (2011): } \\
\text { EBertolucci et al. (2001). }\end{array}$ \\
\hline Br-CAMCOG-R & $\begin{array}{l}\text { Média }^{c} \\
\text { Méc }\end{array}$ & 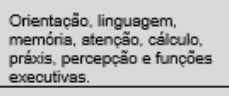 & $\begin{array}{l}60181^{*} \\
83 / 84^{\circ}\end{array}$ & $\begin{array}{l}\text { Alfa de Cronbach: } 0.89^{\circ} \\
\text { CCl: } 0.93^{\circ}\end{array}$ & $\begin{array}{l}88 \% " \\
81.0 \% "\end{array}$ & $\begin{array}{l}84 \% " \\
88.0 \%{ }^{\circ}\end{array}$ & 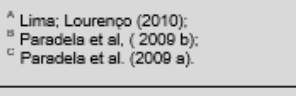 \\
\hline ACE-R & $\begin{array}{l}18 \text { min." } \\
\text { Média }\end{array}$ & $\begin{array}{l}\text { Orientaçäolatenç̃̃o, } \\
\text { memória, fluênciia verbal, } \\
\text { lingugem, habilidade visug- } \\
\text { espaciais. }\end{array}$ & $\begin{array}{r}82^{*} \\
<78^{\circ}\end{array}$ & Alfa de Cronbach: $0,80^{*}$ & $\begin{array}{l}84 \% " \\
82.26 \% \text { " }\end{array}$ & $\begin{array}{l}100 \%{ }^{*} \\
100 \%\end{array}$ & $\begin{array}{l}\text { AMioshi et al. (2006); } \\
{ }^{\circ} \text { Noone (2015): } \\
{ }^{\mathrm{N}} \text { Carvalho (2009). }\end{array}$ \\
\hline ADAS-Cog & 30 a $45 \mathrm{~min}$. & $\begin{array}{l}\text { Memóris, linguagem. } \\
\text { praxias e compreensão de } \\
\text { ordens }\end{array}$ & $\begin{array}{l}\text { "Escolaridgde: } \\
\text { 0 a } 4 \text { anos: } 22,9 \text { pontos } \\
5 \text { a } 11 \text { anos: } 22,4 \text { pontos } \\
12 \text { ou mais: } 15,4 \text { pontos }\end{array}$ & $\begin{array}{l}\text { Alfs de Cronbsch: } 0,91^{2} \\
\text { CCl: } 0,80^{\circ} \text { : } \\
\text { Teste-reteste: } 0,83^{\circ}\end{array}$ & $90 \%{ }^{*}$ & $94.7 \%$ & $\begin{array}{l}\text { AMalloy-Diniz et al. (2013): } \\
\text { "Meyer et al. (1997): } \\
\text { "Chu et al. (2000). }\end{array}$ \\
\hline
\end{tabular}

RC: 22961

Disponível em: https://www.nucleodoconhecimento.com.br/psicologia/vantagens-e$\underline{\text { limitacoes }}$ 
Nota: Escol.: escolaridade; coef.: coeficiente; con.: construtiva; pal./palavr.: palavras; rec.: reconhecimento; evoc.: evocação; prax.: praxia; nom.: nomeação; v./ver.: verbal; Bost.: Boston.

\section{CONSIDERAÇÕES FINAIS}

Após análise do conteúdo desta pesquisa, verificou-se que no Brasil existem inúmeros instrumentos para rastreio cognitivo validados, adaptados e normatizados à população brasileira para $\mathrm{AN}$ de idoso com suspeita de demência. No entanto, além das inúmeras vantagens entre os testes de rastreio cognitivo, existem também limitações características que de certa forma os restringem em determinado contexto. Por isso, é importante ter cautela na utilização desses instrumentos.

Neste sentido, faz-se necessário escolher o instrumento que possa se adequar melhor à demanda específica do avaliado. Além disso, o ideal é que o instrumento apresente acurácia, níveis de corte adequados por idade e/ou escolaridade e fidedignidade aceitável baseada em pesquisas empíricas.

Contudo, os testes psicométricos aplicados isoladamente não determinam uma patologia, ou melhor, um diagnóstico nosológico, mesmo porque o rastreio cognitivo, foco desse trabalho, visa facilitar o direcionamento das investigações específicas de cada domínio cognitivo. Por sua vez, essa informação irá auxiliar e complementar o diagnóstico composto por anamnese e/ou entrevistas bem elaboradas que nortearão a hipótese da suposta enfermidade investigada, possibilitando a AN mais abrangente e, consequentemente, a estruturação do laudo conclusivo.

Por isso, nesse artigo optou-se por identificar e descrever alguns instrumentos de rastreio cognitivo, o que possibilitará a consulta e comparação uns com os outros, de modo a facilitar a seleção daqueles que melhor atenda, ou adeque às necessidades do cliente e do avaliador. Para tanto, considerou-se as características dos domínios investigados de cada instrumento, o tempo de aplicação, o ponto de corte, os escores ou percentis, a confiabilidade, a sensibilidade, a especificidade, além de

RC: 22961

Disponível em: https://www.nucleodoconhecimento.com.br/psicologia/vantagens-elimitacoes 
suas respectivas referências. As características qualitativas das vantagens e limitações de cada teste, escala ou bateria, estão especificados na apresentação dos resultados e discussão.

\section{REFERÊNCIAS}

AMERICAN PSYCHIATRIC ASSOCIATION (APA). DSM-IV. Manual Diagnóstico e Estatístico de Transtornos Mentais. 4. ed. Porto Alegre: ARTMED, 2002.

AMERICAN PSYCHIATRIC ASSOCIATION (APA). DSM-5. Manual Diagnóstico e Estatístico de Transtornos Mentais. Tradução: Maria Inês Corrêa Nascimento 5. ed. Porto Alegre: Artmed, 2014.

APRAHAMIAN, Ivan et al. Rastreio cognitivo em idosos para o clínico. Revista Brasileira Clinica Médica, São Paulo, v. 6, p. 254-259, nov./dez. 2008. Disponível em:< www.sbcm.org.br/revistas/RBCM/RBCM-2008-06.pdf>. Acesso em: 21/10/2016.

; MARTINELLI; YASSUDA. Doença de Alzheimer em idosos com baixa escolaridade: o teste do Desenho do Relógio pode ser útil no rastreio cognitivo? Rev. Soc. Bras. Clín. Méd., São Paulo, v. 6, n. 4, p. 130-134, jul./ago. 2008. Disponível em: <files.bvs.br/upload/S/1679-1010/2008/v6n4/a130-134.pdf>. Acesso em: 18/02/2017.

et al. The Clock Drawing Test. A review of its accuracy in screening for dementia. Dementia \& Neuropsychology, Ribeirão Preto, v. 3, n. 2, p. 74-80, mai/jun. 2009. Disponível em: < http://dx.doi.org/10.1590/S198057642009DN30200002>. Acesso em: 25/05/2017.

ARAÚJO, Cláudia L.O.; NICOLI, Juliana S. Uma revisão bibliográfica das principais demências que acometem a população brasileira. Revista Kairós Gerontologia, 
São Paulo, v.13, n.1, p. 231-244, jun. 2010. Disponível em: < file://C:/Users/Cliente/Downloads/4872-11570-1-SM.pdf>. Acesso em: 10/06/2010.

ATALAIA-SILVA, Kelly C.; LOURENÇO, Roberto A. Tradução, adaptação e validação de construto do Teste do Relógio aplicado entre idosos no Brasil. Revista de Saúde Pública, São Paulo, v. 42, n. 5, p. 930-937, out. 2008. Disponível em:< http://www.revistas.usp.br/rsp/article/viewFile/32517/34806>. Acesso em: 19/05/2017.

BATISTA, Juliana A. et al. Reliability of cognitive tests of ELSA-Brasil, the brazilian longitudinal study of adult health. Dementia \& Neuropsychologia, v.7, n. 4, p. 367373, dez. 2013. Disponível em: <http://www.scielo.br/pdf/dn/v7n4/1980-5764-dn-704-00367.pdf>. Acesso em: 03/01/2017.

BEATO, Rogério G., NITRINI, R, FORMIGONI, AP, CARAMELLI . Brazilian version of the frontal assessment battery (FAB): Preliminary data on administration to healthy elderly. Dementia \& Neuropsychologia, São Paulo, v. 1, p. 59-65, abr. 2007. Disponível em: < http://dx.doi.org/10.1590/S1980-57642008DN10100010>. Acesso em: 23/01/2017.

et al. Frontal assessment battery in a Brazilian sample of healthy controls: normative data. Arquivos de neuropsiquiatria, São Paulo, v.70, n. 4, p. 278-280, fev. 2012. Disponível em:< http://dx.doi.org/10.1590/S0004282X2012005000009>. Acesso em: 15/02/2017.

BERTOLUCCI, Paulo H.F. et al. O Mini-Exame do Estado Mental em uma população geral: impacto da escolaridade. Arq. Neuro-Psiquiatr., São Paulo , v. 52, n. 1, p. 01-07, mar. 1994. Disponível em: <https://doi.org/10.1590/S0004282X1994000100001>. Acesso em: 16/07/2016.

et al. Applicability of the CERAD neuropsychological battery to Brazilian elderly. Arquivos de neuropsiquiatria, São Paulo, v. 59, n. 3A, p. 532- 
536, sept. 2001. Disponível em:< http://dx.doi.org/10.1590/S0004282X2001000400009>. Acesso em: 26/05/2017.

BOTTINO, C.M.C. et al. CAMDEX: The Cambridge Examination for Mental Disorders of the Elderly: tradução e adaptação para o português. Projeto Terceira Idade (PROTER). 1999.

BRUCKI, S.M.D.; ROCHA, M.S.G. Category fluency test: effects of age, gender and education on total scores, clustering and switching in Brazilian Portuguese-speaking subjects. Brazilian journal of medical and biological research, v. $37, \mathrm{n} .12$, p.1771-1777, 2004. Disponível em: <http://dx.doi.org/10.1590/S0100879X2004001200002>. Acesso em: 22/10/2016.

et al. Dados normativos para o teste de fluência verbal categoria animais em nosso meio. Arq. Neuropsiquiatria, São Paulo, v. 55, n. 1, p. 56-61, 1997. Disponível em:< http://dx.doi.org/10.1590/S0004282X1997000100009>. Acesso em: 16/02/2017.

et al. Formal education, health literacy and mini-mental state examination. Dement. Neuropsychology, São Paulo, v. 5, n. 1, p. 26-30, mar. 2011. Disponível em: < http://www.scielo.br/pdf/dn/v5n1/1980-5764-dn-5-01-00026.pdf>. Acesso em: 12/04/2017.

CAIXETA, L.; FERREIRA, S. B. Manual de Neuropsicologia: dos princípios à reabilitação. 2. ed. São Paulo: Atheneu, 2012. 489p.

CARVALHO, Viviane A.; CARAMELLI, Paulo. Brazilian adaptation of the Addenbrooke's cognitive examination-revised (ACE-R). Dement. Neuropsychol., São Paulo, v.1, n.2, p. 212-216, abr./jun. 2007. Disponível em: < http://www.scielo.br/pdf/dn/v1n2/1980-5764-dn-1-02-00212.pdf>. Acesso em: 24/11/2016.

RC: 22961

Disponível em: https://www.nucleodoconhecimento.com.br/psicologia/vantagens-elimitacoes 
ADDENBROOKE'S COGNITIVE EXAMINATIONREVISED (ACE-R): ADAPTAÇÃO TRANSCULTURAL, DADOS NORMATIVOS DE IDOSOS COGNITIVAMENTE SAUDÁVEIS E DE APLICABILIDADE COMO INSTRUMENTO DE AVALIAÇÃO COGNITIVA BREVE PARA PACIENTES COM DOENÇA DE ALZHEIMER PROVÁVEL LEVE. 2009. 89f. Dissertação (Mestrado em Neurologia da Faculdade de Medicina-FM) - Universidade de SP-USP, São Paulo. Disponível em:

file:///C:/Users/Cliente/Downloads/VIVIANEAMARALCARVALHO.pdf >. Acesso em: 22/05/2017.

CECATO, J.F. et al. MoCA predictive power in neuropsychological assessment of patients with dementia. Rev. bras. geriatr. gerontol., Rio de Janeiro , v. 17, n. 4, p. 707-719, dez. 2014. Disponível em:< http://dx.doi.org/10.1590/18099823.2014.13123>. Acesso em: 25/04/2017.

CHAVES, Márcia L.F. et al. Doença de Alzheimer: avaliação cognitiva, comportamental e funcional. Dement. neuropsychol, São Paulo, v. 5, n.1, jun. 2011. Disponível em:< http://www.redalyc.org/html/3395/339529025004/>. Acesso em: 05/09/2016.

$\mathrm{CHU}$, Leung W. et al. The reliability and validity of the Alzheimer's Disease Assessment Scale Cognitive Subscale (ADAS-Cog) among the elderly Chinese in Hong Kong. Annals of the Academy of Medicine, Singapore, v. 29, n. 4, p. 474485, 2000. Disponível em: <http://europepmc.org/abstract/med/11056778>. Acesso em: 20/11/2016.

DA MOTA, Márcia M.P.E. Triagem cognitiva: comparações entre o Mini Mental e o teste de trilhas. Estudos de Psicologia, v.25, n 3, p. 353-359, jul./set. 2008. Disponível em: <http://www.scielo.br/pdf/estpsi/v25n3/a04v25n3>. Acesso em: 04/01/2017. 
DAVIS, Daniel H. J. et al. Montreal Cognitive Assessment for the diagnosis of Alzheimer's disease and other dementias. Cochrane Database of Systematic Reviews., v.10. $\quad$ n. $\quad 2, \quad$ Out. $2015 . \quad$ Disponível em:<http://onlinelibrary.wiley.com/doi/10.1002/14651858.CD010775.pub2/pdf>. Acesso em: 26/02/2017.

DE PAULA, Jonas $\mathrm{J}$. et al. Screening for executive dysfunction with the Frontal Assessment Battery: psychometric properties analysis and representative normative data for Brazilian older adults. Psicologia em Pesquisa, Juiz de Fora, v. 7, n. 1, p. 89-98, jan./jun. 2013. Disponível em:< http://dx.doi.org/10.5327/Z1982$1247201300010010>$. Acesso em: 11/01/2017.

DINIZ, Breno S. et al. Mild cognitive impairment: cognitive screening or neuropsychological assessment? Revista Brasileira de Psiquiatria, São Paulo, v. 30, n. 4, p. 316-321, dez. 2008. Disponível em: < http://dx.doi.org/10.1590/S151644462008000400003>. Acesso em: 18/04/2017.

DUBOIS, B. et al. The FAB A frontal assessment battery at bedside. Neurology, Downey, v. 55, n. 11, p. 1621-1626, set. 2000. Disponível em<. http://dx.doi.org/10.1212/WNL.55.11.1621>. Acesso em: 29/08/2016.

ERZIGKEIT, H. The SKT - A short cognitive performance test as an instrument for the assessment of clinical efficacy of cognition enhancers. In: Diagnosis and treatment of senile dementia. Springer Berlin Heidelberg, p. 164-174, 1989. Disponível em: $<\mathrm{https}: / /$ pageone.live.cf.public.springer.com/pdf/preview/10.1007/978-3-642-46658-8_14>. Acesso em: 17/10/2016.

FILLENBAUM, Gerda G. et al. Norms for CERAD constructional praxis recall. The Clinical neuropsychologist, v. 25, n. 8, p. 1345-1358, out. 2011. Disponível em: <https://www.ncbi.nlm.nih.gov/pmc/articles/PMC3398459/>. Acesso em: 25/06/2016. 
FOSS, Maria P.; VALE, Francisco A.C; SPECIALI, José G. Influência da escolaridade na avaliação neuropsicológica de idosos: Aplicação e análise dos resultados da Escala de Mattis para Avaliação de Demência (Mattis Dementia Rating Scale-MDRS). Arquivos de Neuropsiquiatria, v. 63, n. 1, p. 119-126, 2005. Disponível em: <http://www.scielo.br/pdf/\%0D/anp/v63n1/23610.pdf>. Acesso em 10/11/2016.

et al. Mattis Dementia Rating Scale (DRS): Normative data for the Brazilian middle-age and elderly populations. Dementia \& Neuropsychology, Ribeirão Preto, v. 7, n. 4, p. 374-379, nov. 2013. Disponível em: < http://www.scielo.br/pdf/dn/v7n4/1980-5764-dn-7-04-00374.pdf>. Acesso em: 28/11/2016.

FOLSTEIN, Marshal F.; FOLSTEIN, Susan E.; MCHUGH, Paul R. "Mini-mental state": a practical method for grading the cognitive state of patients for the clinician. Journal of psychiatric research, Grã-Bretanha, v. 12, n. 3, p. 189-198, November 1975.

Disponível em:<http://home.uchicago.edu/ tmurray1/research/articles/printed\%20and\%20read/ mini\%20mental\%20state_a\%20practical\%20method\%20for\%20grading\%20the\%20c ognitive\%20state\%20of\%20patients\%20for\%20the\%20clinician.pdf>. Acesso em: $18 / 08 / 2016$.

FLAKS, Mariana K. TESTE BREVE DE PERFOMANCE COGNITIVA (SKT): ESTUDO DE VALIDAÇÃO E PROPRIEDADES DIAGNÓSTICAS EM UMA AMOSTRA CLÍNICA BRASILEIRA. 2008. 137 f. Tese (Doutorado da Faculdade de Medicina-FM) - Universidade de SP-USP, São Paulo, 2008. Disponível em: <file:///C:/Users/Cliente/Downloads/marianakflaks.pdf>. Acesso em: 03/02/2017.

FREITAS, Sandra; SIMÕES, Mário R. Teste do desenho do relógio: utilidade e validade como instrumento de rastreio cognitivo. Revista de Psicologia, Educação e Cultura, Carvalhos, v. 14, n.2, p.319-338, dez. 2010. Disponível em: $<$ https://www.researchgate.net/profile/Marisa_Avila2/publication/271192990_The_Int

RC: 22961

Disponível em: https://www.nucleodoconhecimento.com.br/psicologia/vantagens-elimitacoes 
erpersonal_Competence_Questionnaire_ICQ_A_study_with_Portuguese_adolescent s/links/54bfa85a0cf28eae4a65fe05.pdf\#page=83>. Acesso em: 26/02/2017.

LIMA, Daniele A.; LOURENCO, Roberto A. Adaptação para o Português da seção A do Cambridge Examination for Mental Disorders of the Elderly-Revised Version (CAMDEX-R) para o diagnóstico de demência. Cad. Saúde Pública, v. 26, n. 7, p. 1345-54, Jul. 2010. Disponível em:< http://dx.doi.org/10.1590/S0102311 X2010000700013 >. Acesso em: 16/03/2017.

LOURENÇO, Roberto A. et al. The Clock Drawing Test: performance among elderly with low educational level. Rev. Bras. Psiquiatr., São Paulo , v. 30, n. 4, p. 309-315, dez. 2008. Disponível em: < http://dx.doi.org/10.1590/S1516-44462008000400002>. Acesso em: 23/11/2016.

; VERAS, R.P.; RIBEIRO, P.C.C. Confiabilidade teste reteste do Mini Exame do Estado Mental em uma população idosa assistida em uma unidade ambulatorial de saúde. Rev. Bras. Geriatr. Gerontol., Rio de Janeiro, v. 11, n. 1 , p. 7-16, jan./ago. 2008. Disponível em: < http://www.redalyc.org/pdf/4038/403838777002.pdf>. Acesso em: 14/05/2016.

MALLOY-DINIZ et al. Avaliação Neuropsicológica. Porto Alegre: Artmed, 2010.

et al. Neuropsicologia do envelhecimento: uma abordagem multidimensional. Porto Alegre: Artmed, 2013. 456 p.

MACHADO, T.H. et al. Dados normativos de idosos saudáveis na prova de fluência verbal fonêmica-FAS. Dement. Neuropsychol, v. 3, n. 1, p. 55-60, 2009. Disponível em: <http://dx.doi.org/10.1590/S1980-57642009DN30100011 >. Acesso em: 13/01/2017.

MARTINELLI J.E.; APRAHAMIAN, I; CACHIONI, M. O teste do desenho do relógio: revisão crítica de seu valor na triagem de demências iniciais em idosos. In: 
Neri AL, Yassuda MS, (Org.) Velhice bem-sucedida: aspectos afetivos e cognitivos. Campinas: Papirus, p.185-203, 2004.

MATIOLI, M.N.P.S.; CARAMELLI, P. Limitations in differentiating vascular dementia from Alzheimer's disease with brief cognitive tests. Arq. Neuro-Psiquiatr., São Paulo, v. 68, n. 2, p. 185-188, abr. 2010. Disponível em:<http://dx.doi.org/10.1590/S0004-282X2010000200006>. Acesso em: 21/03/2017.

\section{MATIOLI, M.N.P.S. ESTUDO COMPARATIVO DO DESEMPENHO EM TESTES} NEUROPSICOLÓGICOS DE PACIENTES COM DIAGNÓSTICO DE DOENÇA DE ALZHEIMER E DEMÊNCIA VASCULAR. 2005. 161f. Tese (Doutorado da Faculdade de Medicina-FM) - Universidade de SP-USP, São Paulo, 2005. Disponível em: <file://C:/Users/Cliente/Downloads/MNPMatioli.pdf>. Acesso em: 23/11/2016.

MATTIS, S. Dementia Rating Scale: DRS: Professional Manual. PAR; 1988.

MELO, Denise M.; BARBOSA, Altemir J.G. O uso do Mini-Exame do Estado Mental em pesquisas com idosos no Brasil: uma revisão sistemática. Ciênc. saúde coletiva, Rio de Janeiro , v. 20, n. 12, p. 3865-3876, dez. 2015 . Disponível em: <http://www.scielo.br/scielo.php?script=sci_arttext\&pid=S1413$81232015001203865 \&$ Ing=en\&nrm=iso >. Acesso em: 15/Dez/2016.

MEMÓRIA, C.M. et al. Brief screening for mild cognitive impairment: validation of the Brazilian version of the Montreal cognitive assessment. International Journal of Geriatric Psychiatry, Manchester, v. 28, n. 1, p. 34-40, Jan. 2013. Disponível em:<https://www.ncbi.nlm.nih.gov/pubmed/22368034 >. Acesso em: 12/01/2017.

MIOSHI, E. et al. The Addenbrooke's Cognitive Examination Revised (ACE-R): a brief cognitive test battery for dementia screening. International journal of geriatric psychiatry, Manchester, v. 21, n. 11, p. 1078-1085, set. 2006. Disponível em: 
<https://pdfs.semanticscholar.org/ccfc/9ac6dac79933eee239b492b1932eabbe6026.p df>. Acesso em: 16/02/2017.

MONTIEL, José M. et al. Testes do desenho do relógio e de fluência verbal: contribuição diagnóstica para o Alzheimer. Psicologia: teoria e prática, São Paulo, v. 16, n. 1, p. 169-180, jan./abr. 2014. Disponível em: < http://pepsic.bvsalud.org/scielo.php?script=sci_arttext\&pid=S151636872014000100014/>. Acesso em: 15/10/2016.

MOMS, J.C. et al. The Consortium to Establish a Registry for Alzheimer's Disease (CERAD). Part I. Clinical and neuropsychological assesment of Alzheimer's disease. Neurology, v. 39, n. 9, p. 1159-1159, 1989. Disponível em: <http://dx.doi.org/10.1212/WNL.39.9.1159>. Acesso em: 27/04/2017.

MOURA, Samara M. CONTRIBUIÇÕES DE QUATRO INSTRUMENTOS DE TRIAGEM PARA O DIAGNÓSTICO DE DÉFICITS COGNITIVOS NO ENVELHECIMENTO NO BRASIL: VALIDADE DE CRITÉRIO E NORMAS DE DESEMPENHO. 2008. 182 f. Dissertação (Mestrado em Psicologia da Faculdade de Ciências Humanas-FCH) - UFMG, Belo Horizonte, 2008. Disponível em: <http://hdl.handle.net/1843/VCSA-7NYHAR>. Acesso em: 07/04/2017.

MCKHANN, G.M. Clinical diagnosis of Alzheimer's disease Report of the NINCDS-ADRDA Work Group under the auspices of Department of Health and Human Services Task Force on Alzheimer's Disease. Neurology, v. 34, n.7, p. 939944, Jul. 1984. Disponível em: <https://www.ncbi.nlm.nih.gov/pubmed/6610841>. Acesso em: 18/10/2016.

et al. The diagnosis of dementia due to Alzheimer's disease: Recommendations from the National Institute on Aging-Alzheimer's Association workgroups on diagnostic guidelines for Alzheimer's disease. Alzheimer's \& dementia, v.7, n. 3, p. 257-262, mai. 2011. Disponível em : 
<http://europepmc.org/backend/ptpmcrender.fcgi?accid=PMC3096735\&blobtype=pdf >. Acesso em: 23/02/2017.

NASREDDINE, Ziad S. et al. The Montreal Cognitive Assessment, MoCA: a brief screening tool for mild cognitive impairment. Journal of the American Geriatrics Society, La Rioja, v. 53, n. 4, p. 695-699, 2005. Disponível em: < https://www.ncbi.nlm.nih.gov/pubmed/15817019 >. Acesso em: 10/12/2016.

NETO, José G. et al. Diagnóstico diferencial das demências. Rev Psiq Clín, v. 32, n. 3, p. 119-30, 2005. Disponível em: <http://dx.doi.org/10.1590/S010160832005000300004>. Acesso em: 10/05/2016.

NITRINI, Ricardo. et al. Brief cognitive battery in the diagnosis of mild Alzheimer's disease in subjects with medium and high levels of education. Dement Neuropsychology, São Paulo, v. 1, n. 1, p. 32-36, mar. 2007. Disponível em: < http://dx.doi.org/10.1590/S1980-57642008DN10100006>. Acesso em: 13/12/2016.

NOONE, Peter. Addenbrooke's Cognitive Examination-III. Oxford Journals Occupational Medicine, Lond., v. 65, n. 5, p. 418 - 420, jul. 2015. Disponível em: <https://doi.org/10.1093/occmed/kqv041>. Acesso em: 22/09/ 2016.

ORGANIZAÇÃO MUNDIAL DA SAÚDE. CLASSIFICAÇÃO ESTATÍSTICA INTERNACIONAL DE DOENÇAS E PROBLEMAS RELACIONADOS À SAÚDE: CID 10. 10a. ed. São Paulo: Edusp; 1998.

PARADELA, Emylucy M. P.; LOPES, Claudia S.; LOURENÇO, Roberto A. Reliability of the Brazilian version of the Cambridge Cognitive Examination Revised CAMCOGR. Arquivos de neuropsiquiatria, São Paulo, v. 67, n. 2b, p. 439-444, jun. 2009. Disponível em: <http://dx.doi.org/10.1590/S0004-282X2009000300013>. Acesso em: $18 / 08 / 2016$. 
; LOURENÇO, R. A. Is the Cambridge Cognitive ExaminationRevised a good tool for detection of dementia in illiterate Brazilian older adults? Geriatrics \& gerontology international, Main Street, v. 14, n. 4, p. 763-768, out. 2014. Disponível em: <http://onlinelibrary.wiley.com/doi/10.1111/ggi.12161/full>. Acesso em: 10/02/2017.

; LOPES, Claudia S.; LOURENÇO, Roberto A. Adaptação para

o português do Cambridge Cognitive Examination - Revised aplicado em um ambulatório público de geriatria. Caderno de saúde pública, Rio de Janeiro, v. 25, n.12a, p. 2562-2570, dez. 2009. Disponível em: <http://dx.doi.org/10.1590/S0102311X2009001200004>. Acesso em: 26/02/2017.

PORTO, Claudia S. et al. Brazilian version of the Mattis dementia rating scale: diagnosis of mild dementia in Alzheimer's disease. Arquivos de neuropsiquiatria, São Paulo, v. 61, n. 2B, p. 339-345, jun. 2003. Disponível em:<http://dx.doi.org/10.1590/S0004-282X2003000300004>. Acesso em: 28/01/2017.

\section{A ESCALA DE AVALIAÇÃO DE DEMÊNCIA (DRS) NO} DIAGNÓSTICO DE COMPROMETIMENTO COGNITIVO LEVE E DOENÇA DE ALZHEIMER. 2006. 93 f. Tese (Doutorado em Neurologia da Faculdade de Medicina-FM)-Universidade de SP-USP, São Paulo, 2006. Disponível em: <file://C:/Users/Cliente/Downloads/claudiasellittoporto.pdf>. Acesso em: 15/10/2016.

;CARAMELLI, Paulo; NITRINI, Ricardo. The Dementia Rating Scale (DRS) in the diagnosis of vascular dementia. Dementia \& Neuropsychology, Ribeirão Preto, v. 1, n. 3, p. 282-287, jul./set. 2007. Disponível em: $<$ http://www.scielo.br/pdf/dn/v1n3/1980-5764-dn-1-03-00282.pdf >. Acesso em: 11/12/2016.

RAHMAN, T.T.A.; GAAFARY, E.I.; MOHAMED, M. Montreal Cognitive Assessment Arabic version: reliability and validity prevalence of mild cognitive impairment among

RC: 22961

Disponível em: https://www.nucleodoconhecimento.com.br/psicologia/vantagens-e$\underline{\text { limitacoes }}$ 
elderly attending geriatric clubs in Cairo. Geriatrics \& Gerontology International, Chichester, v. 9, n. 1, p. 54-61, February 2009. Disponível em: <https://www.ncbi.nlm.nih.gov/pubmed/19260980 >. Acesso em: 25/11/2016.

RIBEIRO, Pricila C.C. et al. Desempenho de idosos na bateria cognitiva CERAD: relações com variáveis sociodemográficas e saúde percebida. Psicologia: Reflexão e Crítica, Porto Alegre, v. 23, n. 1, p. 102-109, abr. 2010. Disponível em: <http://www.scielo.br/pdf/prc/v23n1/a13v23n1>. Acesso em: 19/03/2017.

ROALF, David R. et al. Comparative accuracies of two common screening instruments for classification of Alzheimer's disease, mild cognitive impairment, and healthy aging. Alzheimer's \& Dementia, New York, v. 9, n. 5, p. 529-537, september 2013. Disponível em: < https://www.ncbi.nlm.nih.gov/pmc/articles/PMC4036230/ >. Acesso em: 14/12/2016.

SANTOS, Cássia S. et al. Avaliação da confiabilidade do Mini Exame do estado mental em idosos e associação com variáveis sociodemográfica. Cogitare enferm, Curitiba, v. $15, \quad$ n. 3 , p. 406-12, jul./set. 2010. Disponível em: <http://www.redalyc.org/html/4836/483648972002/ >. Acesso em: 19/02/2017.

\section{SARMENTO, Ana L.R. APRESENTAÇÃO E APLICABILIDADE DA VERSÃO} BRASILEIRA DA MONTREAL COGNITIVE ASSESSMENT (MOCA) PARA RASTREIO DE COMPROMETIMENTO COGNITIVO LEVE. 2009. 62 fl. Dissertação (Mestrado em Ciências da Escola Paulista de Medicina) - Universidade Federal de São Paulo -USP. São Paulo 2009. Disponível em: <http://vml029.epm.br/bitstream/handle/11600/8967/Publico-00377.pdf?sequence=1 >. Acesso em: 19/01/2017.

SILVA, Ana E.V.F. et al. Importância da escolaridade no rastreio de déficit cognitivo pelo teste do relógio de Shulman. João Pessoa: Departamento de Medicina Interna .Centro de Ciências Médicas, v. 2, n.1, set. 2015. Disponível em: 
<http://www.editorarealize.com.br/revistas/cieh/trabalhos/TRABALHO_EV040_MD2_ SA3_ID1861_17072015203914.pdf>. Acesso em: 08/09/2016.

SILVA, Thais B.L. et al. Fluência verbal e variáveis sociodemográficas no processo de envelhecimento: um estudo epidemiológico. Psicologia: Reflexão e Crítica, Porto Alegre, v. 24, n. 4, p. 739-746, 2011. Disponível em: <http://www.scielo.br/pdf/prc/v24n4/a14v24n4>. Acesso em 20/01/2017.

SLACHEVSKY, Andrea. et al. Frontal assessment battery and differential diagnosis of frontotemporal dementia and Alzheimer disease. Archives of Neurology, Chicago, v. 61, n. 7, p. 1104-1107, july 2004. Disponível em: <file://C:/Users/Cliente/Downloads/NOC30105.pdf >. Acesso em: 29/08/2016.

SCHAFER, Kimberly A. et al. Reliability of Monitoring the Clinical Dementia Rating in Multicenter Clinical Trials. Alzheimer disease and associated disorders, New York, v.18, n. 4, p. 219-222, Oct./Dec. 2004. Disponível em: < https://www.ncbi.nlm.nih.gov/pmc/articles/PMC4367865/ >. Acesso em: 10/01/2017.

SCHULTZ, R.R.; SIVIERO, M.O.; BERTOLUCCI, P.H.F. The cognitive subscale of the" Alzheimer's Disease Assessment Scale" in a Brazilian sample. Brazilian Journal of Medical and Biological Research, Ribeirão Preto, v. 34, n. 10, p. 1295-1302, oct. 2001. Disponível em: <http://dx.doi.org/10.1590/S0100-879X2001001000009>. Acesso em: 12/03/2017.

SUNDERLAND, Trey. et al. Clock drawing in Alzheimer's disease. Journal of the American Geriatrics Society, v. 37, n. 8, p. 725-729, aug 1989. Disponível em: Disponível em: <http://onlinelibrary.wiley.com/doi/10.1111/j.15325415.1989.tb02233.x/full>. Acesso em: 17/02/2017.

SHULMAN, Kenneth I. Clock-drawing: Is it the ideal cognitive screening test? International Journal of Ger iatri Psychiatry, v. 15, n. 6, p. 548-561, jun 2000. Disponível em: $\quad$ http://onlinelibrary.wiley.com/doi/10.1002/1099- 
1166(200006)15:6\%3C548::AID-GPS242\%3E3.0.CO;2-U/full>. Acesso em: 17/ $01 / 2017$.

WEYER, Geerd et al. Alzheimer's Disease Assessment Scale: reliability and validity in a multicenter clinical trial. International Psychogeriatrics, Munieh, v. 9, n. 02, p. 123-138, June 1997. Disponível em:<https://www.ncbi.nlm.nih.gov/pubmed/9309486 >. Acesso em: 12/10/216.

1. O DSM-5 mudou a nomenclatura para Transtornos Neurocognitivos (TNCs) o que era caracterizado no DSM-IV como Demência, Delirium, Transtorno Amnéstico e Outros Transtornos Cognitivos. A demência está incorporada à entidade recém-nomeada transtorno neurocognitivo maior, embora não esteja excluído o uso do termo demência em subtipos etiológicos nos quais é um termo-padrão. Além disso, o DSM-5 reconhece um nível menos grave de prejuízo cognitivo, o transtorno neurocognitivo leve, que pode também ser foco de cuidado, e que, no DSM-IV, era parte de "Transtorno Cognitivo Sem Outra Especificação" e que também é conhecido pelo termo Comprometimento Cognitivo Leve (CCL). Há critérios diagnósticos para ambas as entidades sindrômicas, seguidos de critérios diagnósticos para os diferentes subtipos etiológicos. Vários TNCs costumam coexistir, e suas relações podem ser ainda mais bem caracterizadas sob diferentes subtítulos incluindo "Diagnóstico Diferencial" (p. ex., TNC devido à DA versus TNC vascular), "Fatores de Risco e Prognóstico" (p. ex., patologia vascular que aumenta a expressão clínica da DA) e/ou "Comorbidade" (p. ex., patologia mista de DA-vascular). O termo demência é mantido no DSM-5 para continuidade, podendo ser usado em contextos em que médicos e pacientes estejam acostumados a ele. Embora demência seja o termo habitual para transtornos como as demências degenerativas, que costumam afetar adultos com mais idade, o termo transtorno neurocognitivo é amplamente empregado, sendo, em geral, o termo preferido para condições que afetam pessoas mais jovens, como o prejuízo secundário a lesão cerebral traumática ou a infecção 
pelo HIV. A definição de TNC maior, além disso, é mais ampla que o termo demência, no sentido de que pessoas com declínio substancial em um só domínio podem receber esse diagnóstico, mais notadamente a categoria do DSM-IV "Transtorno Amnéstico", agora diagnosticado como TNC maior devido a outra condição médica e para o qual o termo demência não será utilizado (APA, 2014).

Enviado: Dezembro, 2017

Aprovado: Novembro, 2018 